TRANSACTIONS OF THE

AMERICAN MATHEMATICAL SOCIETY

Volume 289, Number 1, May 1985

\title{
DEGREES OF INDISCERNIBLES IN DECIDABLE MODELS
}

\author{
BY \\ H. A. KIERSTEAD ${ }^{1}$ AND J. B. REMMEL ${ }^{2}$
}

\begin{abstract}
We show that the problem of finding an infinite set of indiscernibles in an arbitrary decidable model of a first order theory is essentially equivalent to the problem of finding an infinite path through a recursive $\omega$ branching tree. Similarly, we show that the problem of finding an infinite set of indiscernibles in a decidable model of an $\omega$-categorical theory with decidable atoms is essentially equivalent to finding an infinite path through a recursive binary tree.
\end{abstract}

Introduction. Ehrenfeucht and Mostowski [2] introduced the notion of indiscernibles and proved that every first order theory has a model with an infinite set of order indiscernibles. In [6], we investigated the question of which decidable theories have decidable models with infinite recursive sets of indiscernibles. For example, we showed that every $\omega$-stable decidable theory and every stable theory which has a certain strong decidability property called $\exists Q$-decidability have such models. Moreover, we gave a series of examples of decidable theories which have no decidable models with infinite recursive sets of indiscernibles which show that the various hypotheses of our positive results are necessary.

In this paper, we investigate the possible degrees of indiscernibles in a decidable model $\mathcal{M}$. We shall show, in a sense to be made precise in $\S 1$, that the problem of finding a set of indiscernibles in an arbitrary decidable model $\mathcal{M}$ of a first order theory $T$ is essentially equivalent to the problem of finding an infinite path through a recursive $\omega$-branching tree $\tau$. Similarly, we shall show that the problem of finding an infinite set of indiscernibles in a decidable model $\mathcal{M}$ of an $\omega$-categorical theory $T$ with decidable atoms is essentially equivalent to finding an infinite path through a recursive binary tree $\tau$. Then since the possible degrees of infinite paths through recursive trees have been extensively studied in the literature, we are able to derive a number of corollaries about the degrees of indiscernibles in decidable models. For example, we shall show there is a decidable theory $T$ for which every decidable model $\mathcal{M}$ of $T$ has an infinite set of indiscernibles but that no decidable model $\mathcal{M}$ of $T$ has an infinite set of indiscernibles $I$ which is hyperarithmetic. In contrast, we will show that every $\omega$-categorical decidable model $\mathcal{M}$ has an infinite set of indiscernibles which is recursive in $\mathbf{Q}^{\prime}$, the jump of the degree of the recursive sets. Also we shall show that the question of whether an arbitrary decidable model $\mathcal{M}$ has an infinite set of indiscernibles is a complete $\Sigma_{1}^{1}$ question.

Received by the editors September 6, 1983.

1980 Mathematics Subject Classification. Primary 03B25.

${ }^{1}$ The first author was partially supported by N.S.F. Grant \# ISP-8011451.

${ }^{2}$ The second author was partially supported by N.S.F. Grant \# MCS 82-02333. 
In $\S 1$, we shall deal with preliminaries, give a precise definition of what we mean for the problem of finding indiscernibles in a decidable model $\mathcal{M}$ to be equivalent to finding a path through a recursive tree $\tau$, and recall some basic model-theoretic lemmas from [6]. In $\S 2$, we shall investigate the degrees of indiscernibles in arbitrary decidable models and in $\S 3$, we shall investigate the degrees of indiscernibles in $\omega$ categorical decidable models.

Finally, we would like to thank V. Harnik for his careful reading of this paper and our previous paper [6] and for his helpful suggestions.

1. Definitions, notations and preliminaries. $L$ will be a first order language. $T$ will be a countable theory over $L$ which has an infinite model. The (possibly indexed) letter $\mathcal{M}$ will represent a countable model of $T$ and we will usually not distinguish between $\mathcal{M}$ and its domain $\operatorname{do}(\mathcal{M})$. Letters $A, B, C$, etc. will usually denote countable subsets of $\mathcal{M}$. Finite sequences $a_{1}, \ldots, a_{n}$ will be represented by $\bar{a}$. We shall write $\bar{a} \in A$ when we really mean that each element of the range of $\bar{a}$ is in $A . L(A)$ is the language formed by adding new constant symbols for the elements of $A$ to $L$. The type of $\bar{a} \in \mathcal{M}$ over $B$ is the set of formulas $t(\bar{a} / B)=\{\rho(\bar{x}) \in L(B) \mid \mathcal{M} \models \rho(\bar{a})\}$. An atomic type is the restriction of a type to atomic and negations of atomic formulas. A model $\mathcal{M}$ is atomicly homogeneous if and only if whenever $\bar{a}, c, \bar{b} \in \mathcal{M}$ are such that $\bar{a}$ and $\bar{b}$ realize the same atomic type, there exists $d \in \mathcal{M}$ such that $(\bar{a}, c)$ and $(\bar{b}, d)$ realize the same atomic types. Unless stated otherwise, we shall assume that the domain of a decidable model $\mathcal{M}$ is the set of natural numbers $\omega$. A model $\mathcal{M}$ is decidable if the theory of $(\mathcal{M}, m)_{m \in \mathcal{M}}$ is decidable. A decidable theory $T$ has decidable atoms if there is an effective procedure to decide if a formula $\rho\left(x_{1}, \ldots, x_{n}\right)$ is an atom in the Lindenbaum algebra of formulas with $n$ free variables over $T, B_{n}(T)$, for any $n \in \omega$.

The set of finite sequences from $\omega$ will be denoted by $\omega^{<\omega}$ and the set of all finite sequences of 0's and 1's will be denoted by $2^{<\omega} . \phi$ will denote the empty sequence in $\omega^{<\omega}$ as well as the empty set. If $\bar{a}$ and $\bar{b}$ are in $\omega^{<\omega}$, then we write $\bar{a} \subseteq \bar{b}$ if $\bar{a}$ is an initial subsequence of $\bar{b}$. We assume that we have an effective Gödel numbering of $\omega^{<\omega}$ and \# $\left\langle a_{1}, \ldots, a_{n}\right\rangle$ will denote the Gödel number of the sequence $\left\langle a_{1}, \ldots, a_{n}\right\rangle . I=\left\langle i_{n}: n \in \omega\right\rangle$ is a sequence of indiscernibles iff $i_{n} \neq i_{m}$ for $m \neq n$ and $t\left(i_{0}, \ldots, i_{n}\right)=t\left(i_{\sigma(0)}, \ldots, i_{\sigma(n)}\right)$ whenever $\sigma$ is a strictly increasing function and $n \in \omega$. (For emphasis, $I$ is sometimes referred to as a set of order indiscernibles.) Now if $I=\left\langle i_{n}: n \in \omega\right\rangle$ is a sequence of indiscernibles in a decidable model $\mathcal{M}$, then we shall often identify $I$ with the set $\left\{\#\left\langle i_{0}, \ldots, i_{n}\right\rangle \mid n \in \omega\right\}$ so that when we talk of the degree of $I$ we mean the Turing degree of $\left\{\#\left\langle i_{0}, \ldots, i_{n}\right\rangle \mid n \in \omega\right\}$. $I(\mathcal{M})$ will denote the set of infinite sequences of indiscernibles in a model $\mathcal{M}$.

An $\omega$-branching tree is a set $\tau \subseteq \omega^{<\omega}$ such that (i) $\phi \in \tau$ and (ii) whenever $\left\langle a_{0}, \ldots, a_{n}\right\rangle \in \tau$, then $\left\langle a_{0}, \ldots, a_{i}\right\rangle \in \mathcal{T}$ for all $i<n . \pi=\left\langle a_{i}: i \in \omega\right\rangle$ is an infinite path through $T$ if $\left\langle a_{0}, \ldots, a_{n}\right\rangle \in \mathcal{T}$ for all $n \in \omega$. Again we shall often identify $\pi$ with the set $\left\{\#\left\langle a_{0}, \ldots, a_{n}\right\rangle \mid n \in \omega\right\}$ so that the degree of $\pi$ is just the Turing degree of the set $\left\{\#\left\langle a_{0}, \ldots, a_{n}\right\rangle \mid n \in \omega\right\}$. The set of infinite paths through $T$ will be denoted by $P(\tau)$. A binary tree $\tau$ is just an $\omega$-branching tree which is contained in $2^{<\omega}$. An $\omega$-branching tree $T$ is recursive if the set of Gödel numbers of elements of $\tau$ is a recursive set. 
Next we want to discuss what it should mean for the problems of finding infinite paths through a recursive $\omega$-branching tree and of finding infinite sets of indiscernibles in a decidable model to be equivalent. Intuitively, it should be that for any given decidable model $\mathcal{M}$, there is a recursive $\omega$-branching tree $\tau_{\mathcal{M}}$ such that there is an effective correspondence between $I(\mathcal{M})$ and $P\left(\tau_{\mathcal{M}}\right)$ and similarly for any given $\omega$-branching tree $\tau$, there is a decidable model $\mathcal{M}_{\tau}$ such that there is an effective correspondence between $P(\tau)$ and $I\left(\mathcal{M}_{\tau}\right)$. However, such a requirement is too strong. That is, there are recursive $\omega$-branching trees such that $\operatorname{card}(P(\tau))=1$ while clearly if $I(\mathcal{M}) \neq \phi$ for a decidable model $\mathcal{M}$, then since every subsequence of a sequence of indiscernibles is a sequence of indiscernibles, $\operatorname{card}(I(\mathcal{M}))=2^{\omega}$. Thus, in general, we cannot expect an effective $1: 1$ correspondence between $P(\tau)$ and $I\left(\mathcal{M}_{\tau}\right)$ as above. But nevertheless there is a natural weaker notion of a correspondence between $P(\tau)$ and $I(\mathcal{M})$ for some fixed recursive $\omega$-branching tree $T$ and decidable model $\mathcal{M}$. Let $\chi_{e}^{A}(x)$ denote the output of the eth Turing oracle machine with oracle $A \subseteq \omega$ started on input $x$. We shall write $\chi_{e}^{A}=B$ if for all $x \in \omega$,

$$
\chi_{e}^{A}(x)= \begin{cases}0 & \text { if } x \notin B, \\ 1 & \text { if } x \in B .\end{cases}
$$

then we say that the problem of finding an infinite path through $\tau$ is effectively equivalent to the problem of finding an infinite sequence of indiscernibles in $\mathcal{M}$, written $P(\tau) \approx I(\mathcal{M})$, if there exist $e$ and $f$ such that

(i) if $I \in I(\mathcal{M})$, then $\chi_{e}^{I}=\pi_{I} \in P(T)$,

(ii) if $\pi \in P(\tau)$, then $\chi_{f}^{\pi}=I_{\pi} \in I(\mathcal{M})$, and

(iii) for all $\pi \in P(\tau)$, if $\chi_{f}^{\pi}=I_{\pi}$, then $\chi_{e}^{I_{\pi}}=\pi$.

Note that by clauses (ii) and (iii) of the definition of $P(\tau) \approx I(\mathcal{M})$, it follows that $\operatorname{deg}(\pi)=\operatorname{deg}\left(I_{\pi}\right)$ for all $\pi \in P(\tau)$, where for any $A \subseteq \omega, \operatorname{deg}(A)$ denotes the Turing degree of $A$. Moreover by clause (i), we have that for each $I \in I(\mathcal{M})$, $\operatorname{deg}\left(\pi_{I}\right) \leq_{T} \operatorname{deg}(I)$, where $\leq_{T}$ denotes Turing reducibility.

Now in $\S 2$, we shall show that for every recursive $\omega$-branching tree $\tau$, there is a decidable model $\mathcal{M}_{\tau}$ such that $P(\tau) \approx I\left(M_{\tau}\right)$. Thus, the problem of finding infinite sequences of indiscernibles in $\mathcal{M}_{\tau}$ is at least as complex as finding an infinite path through $\tau$. Similarly, we show that for each decidable model $\mathcal{M}$, there is a recursive $\omega$-branching $\tau_{\mathcal{M}}$ such that $P\left(\tau_{\mathcal{M}}\right) \approx I(\mathcal{M})$ so that from any infinite path $\pi$ through $\tau_{\mathcal{M}}$, we can construct an infinite sequence of indescernibles in $\mathcal{M}$. In $\S 3$, we shall give similar correspondences between decidable $\omega$-categorical models of decidable theories $T$ with decidable atoms and recursive binary trees.

We end this section by recalling some basic definitions and lemmas from [6] on which most of our constructions in $\S \S 2$ and 3 are based. Lemmas 1.2 and 1.3 below correspond to Lemmas 2.3 and 2.4 of [6], respectively. However, Lemma 2.4 of [6] was stated incorrectly and without proof. In Lemma 1.3, we provide the corrected version of Lemma 2.4 of [6] and give the proof. We note, however, that each of the constructions of [6] which relied on this lemma are not affected by the correction.

Let $K$ be a class of finite structures over a finite language $L$ which has only relation symbols. We shall assume that if $A \in K$, then $A \subseteq \omega$. If $f$ is any $1: 1$ map from $A$ into $\omega, f[A]$ is the structure whose domain is the range of $f$ and is such that $f[A] \models R(\bar{x})$ iff $A \models R\left(f^{-1}(\bar{x})\right)$, where $R$ is any relation symbol of $L$. 
DEFINITION 1.1. (i) $K$ is closed under isomorphisms iff whenever $A \in K$ and $f: A \rightarrow \omega$ is $1: 1$, then $f[A] \in K$.

(ii) $K$ has the strong amalgamation property iff whenever $A, B \in K$ are such that $A|(A \cap B)=B|(A \cap B)$, then there exists $D \in K$ such that $A, B \subseteq D$.

(iii) $K$ is said to be a full family of structures iff $K$ is closed under isomorphisms, $K$ has the strong amalgamation property, and $K$ is closed under substructures.

In what follows $Q x$ is the quantifier which asserts "there exist infinitely many". A theory $T$ is said to be $\mathcal{L}(Q)$-decidable iff there is an effective procedure which decides for any $\psi \in \mathcal{L}(Q)$ whether $T \cup\{\psi\}$ has a model.

LEMMA 1.2. Let $K$ be a full family of structures which is recursive, i.e., given any finite structure $A$ over $L$ with $A \subseteq \omega$, we can effectively decide if $A \in K$. Then:

(a) there is a decidable atomicly homogeneous model $\mathcal{M}$ such that

(i) every finite substructure of $\mathcal{M}$ is in $K$, and

(ii) any structure in $K$ is embeddable in $\mathcal{M}$;

(b) any two atomicly homogeneous structures satisfying (i) and (ii) are isomorphic;

(c) $T$, the theory of $\mathcal{M}$, is an $\omega$-categorical decidable theory which admits the elimination of quantifiers;

(d) for any formula $\rho(x \bar{y}) \in L$,

$$
T \vdash(\forall \bar{y})\left[(\exists x)(\rho(x \bar{y})) \wedge \bigwedge_{y_{i} \in \bar{y}} x \neq y_{i} \rightarrow(Q x)(\rho(x \bar{y}))\right]
$$

and thus $T$ is $L(Q)$-decidable.

LEMMA 1.3. Let $L_{0} \subseteq L_{1} \subseteq L_{2} \subseteq \cdots$ be an effective sequence of finite languages with only relation symbols. Let $K_{0}, K_{1}, K_{2}, \ldots$ be an effective sequence of classes of finite structures such that for each $i$ :

(a) $K_{i}$ is a recursive full family of finite structures over $L_{i}$;

(b) for each $i<j$ and $A \in K_{j}, A \mid L_{i} \in K_{i}$;

(c) for each $i<j, B^{*} \in K_{j}$ and $A \in K_{i}$, if $B^{*} \mid L_{i}$ is a submodel of $A$,

then $A$ can be expanded to a structure $A^{*} \in K_{j}$ such that $B^{*}$ is a submodel of $A^{*}$.

Let $T_{i}$ be the $\omega$-categorical, $L_{i}(Q)$-decidable theory constructed from $K_{i}$ as in Lemma 1.2. Then $T=\bigcup_{i \in \omega} T_{i}$ is a consistent, complete, $L(Q)$-decidable theory which admits the elimination of quantifiers, where $L=\bigcup_{i \in \omega} L_{i}$.

PROOF. It suffices to show that for all $i<j, T_{i} \subset T_{j}$. We shall accomplish this by proving that the restriction of the atomicly homogeneous model $A_{j}$ of $T_{j}$ to $L_{i}$ is isomorphic to the atomicly homogeneous model $A_{i}$ of $T_{i}$. We do this by checking that (1) $A_{j} \mid L_{i}$ and $A_{i}$ realize the same atomic types over $L_{i}$, and (2) $A_{j} \mid L_{i}$ is atomicly homogeneous over $L_{i}$. Suppose the atomic type $p$ is realized by $\bar{a}$ in $A_{i}$. Let $A=A_{i}\left\lceil\bar{a}\right.$. By Lemma $1.2, A \in K_{i}$. Let $B^{*}=\varnothing$. Then $B^{*} \in K_{j}$ and $B^{*} \mid L_{i} \subset A$. Thus by (c), $A$ can be expanded to $A^{*} \in K_{j}$; by Lemma $1.2, A^{*}$ can be embedded into $A_{j}$; so $p$ is realized by $A_{j}$. An even easier argument using (b) shows that if $p$ is an atomic type over $L_{i}$ realized by $A_{j} \mid L_{i}$, then $p$ is realized by $A_{i}$. Finally we show that $A_{j} \mid L_{i}$ is atomicly homogeneous. Let $\bar{a}, \bar{b}, c \in A_{j}$ be such that the atomic type of $\bar{a}$ over $L_{i}$ is the same as that of $\bar{b}$. By the atomic homogeneity of $A_{j}$ it suffices to find $\bar{a}^{\prime}, \bar{b}^{\prime}, c^{\prime}, d^{\prime} \in A_{j}$ such that $(*)$ the atomic type of $\bar{a}^{\wedge} \bar{b}^{\wedge} c$ 
over $L_{j}$ is the same as that of $\bar{a}^{\prime \wedge} \bar{b}^{\prime \wedge} c^{\prime}$ and the atomic type of $\bar{a}^{\prime `} c^{\prime}$ over $L_{i}$ is the same as that of $\bar{b}^{\prime \wedge} d^{\prime}$. Let $B^{*}=A_{j} \mid \bar{a}^{\wedge} \bar{b}^{\wedge} \bar{c}$ and $B=B^{*} \mid L_{i}$. Then $B^{*} \in K_{j}$ and by (b), $B \in K_{i}$. Since $K_{i}$ is closed under submodels, $C=B \uparrow^{\wedge} \bar{a}^{\wedge} \in K_{i}$. Since $K_{i}$ is closed under isomorphisms there exists $D \in K_{i}$ such that $\operatorname{do}(D)=\bar{b}^{\wedge} d$ and $C$ is isomorphic to $D$ by a map that sends $\bar{a}^{\wedge} c$ to $\bar{b}^{\wedge} d$. Since $K_{i}$ has the amalgamation property there exists $A \in K_{i}$ such that $B$ and $D$ are submodels of $A$. By (c), $A$ can be expanded to $A^{*} \in K_{j}$ so that $B^{*} \subset A^{*}$. Thus $A^{*}$ can be embedded into $A_{j}$, with image $\bar{a}^{\prime \wedge} \bar{b}^{\prime} \backsim c^{\prime} d^{\prime}$ which satisfies $(*)$.

As previously noted, Lemma 2.4 of [6], the lemma of [6] which corresponds to Lemma 1.3 above, was incorrectly stated. The problem was that in Lemma 2.4 of [6], condition (c) of Lemma 1.3 was replaced by the weaker condition: $\left(c^{*}\right)$ for each $i<j$ and $A_{i} \in K_{i}, A_{i}$ can be expanded to a structure in $K_{j}$. Unfortunately condition ( $\mathrm{c}^{*}$ ) is not enough to guarantee the atomic homogenity of $A_{j}\left\lceil L_{i}\right.$ which is used in our proof of Lemma 1.3. However, the two theories in Examples 2.6 and 2.7 of [6] whose constructions were based on Lemma 2.4 of [6] continue to have the properties claimed there as it is easy to check that in those constructions, the stronger condition (c) of Lemma 1.3 holds.

2. Indiscernibles in arbitrary decidable models. In this section we shall give two correspondences which will show that the problem of finding infinite sets of order indiscernibles in decidable models is essentially equivalent to the problem of finding an infinite path through an $\omega$-branching recursive tree, and then derive a number of corollaries from the two correspondences.

First, it is rather trivial to show that for any given decidable model $\mathcal{M}$ over a first order language $L$, there exists a recursive $\omega$-branching tree $\tau_{\mathcal{M}}$ such that there is an effective $1: 1$ correspondence between $I(\mathcal{M})$ and $P\left(\tau_{\mathcal{M}}\right)$. That is, let $\rho_{0}, \rho_{1}, \ldots$ be an effective list of all formulas of $L$ such that the free variables of $\rho_{i}$ are among $x_{0}, \ldots, x_{i}$. We say that a sequence $\bar{a}=a_{0}, \ldots, a_{n}$ from $\mathcal{M}$ is a sequence of indiscernibles with respect to $\rho_{0}, \ldots, \rho_{n}$ over $\mathcal{M}$ if for all $i \leq n$ : $\mathcal{M} \models$ $\rho_{i}\left(a_{0}, \ldots, a_{k-1}\right) \Leftrightarrow \rho_{i}\left(a_{\sigma(0)}, \ldots, a_{\sigma(k-1)}\right)$ for all increasing maps $\sigma:\{0, \ldots, k-1\} \rightarrow$ $\{0, \ldots, n\}$, where $\rho_{i}$ has exactly $k$ free variables. Then we simply let $\tau_{\mathcal{M}}$ be the recursive $\omega$-branching tree such that $\phi \in \tau_{\mathcal{M}}$ and $a_{0}, \ldots, a_{n} \in \tau_{\mathcal{M}}$ iff $a_{0}, \ldots, a_{n} \in \mathcal{M}$, and $a_{0}, \ldots, a_{n}$ is a sequence of indiscernibles with respect to $\rho_{0}, \ldots, \rho_{n}$ over $\mathcal{M}$. It is then clear that an infinite path $\pi=\left\langle a_{n}: n \in \omega\right\rangle$ through $\tau$ is just an infinite set of order indiscernibles in $\mathcal{M}$ and vice versa. Thus we have the following.

Proposition 2.1. For any decidable model $\mathcal{M}$, there exists a recursive $\omega$ branching tree $\tau_{\mathcal{M}}$ such that $P\left(\tau_{\mathcal{M}}\right) \approx I(\mathcal{M})$.

An immediate consequence of Proposition 2.1 is that if $\mathcal{M}$ is a decidable model which has an infinite set of order indiscernibles, then there is a set of indiscernibles in $\mathcal{M}$ recursive in Kleene's 0 . In fact, by the Gandy Basis Theorem (see Theorem XLIII of [8]), we have the following

COROLlaRY 2.2. If $\mathcal{M}$ is a decidable model such that $I(\mathcal{M}) \neq \varnothing$, then there exists an infinite set of order indiscernibles $I$ in $\mathcal{M}$ such that the hyperdegree of $I$ is strictly less than the hyperdegree of 0 .

Similarly, if we apply the Harrington-Kechris basis theorem [3], we have the following corollary. 
COROLlaRY 2.3. If $\mathcal{M}$ is a decidable model such that every hyperarithmetic real is recursive in some member of $I(\mathcal{M})$, then $I(\mathcal{M})$ contains elements in every Turing degree above 0 .

Next we turn to the more diticult task of showing that for every recursive $\omega$ branching tree, there exists a decidable model $\mathcal{M}_{\tau}$ such that the problem of finding infinite paths through $\tau$ is effectively equivalent to the problem of finding infinite sets of order indiscernibles in $\mathcal{M}$. In fact, we prove something much stronger.

THEOREM 2.4. Let $\tau$ be a recursive $\omega$-branching tree. Then there exists a complete decidable theory $T$ such that for any decidable model $\mathcal{M}$ of $T, P(T) \approx$ $I(\mathcal{M})$.

ProOF. We shall use Lemma 1.3 to construct our theory $T$. Thus we must specify an increasing sequence of languages, $L_{1} \subseteq L_{2} \subseteq \cdots$, with only relation symbols and an effective sequence of classes of finite structures, $K_{1}, K_{2}, \ldots$, satisfying conditions (a)-(c) of Lemma 1.3 so that if $T_{i}$ is the $\omega$-categorical $L_{i}(Q)$-decidable theory constructed from $K_{i}$ via Lemma 1.2 , then the theory $T=\bigcup_{i=1}^{\infty} T_{i}$ over the language $L=\bigcup_{i=1}^{\infty} L_{i}$ is our desired decidable theory. Before giving the exact definitions of the $L_{i}$ and $K_{i}$, we shall make a few remarks about the basic strategy of our construction. Our final language will contain $|\sigma|+1$-ary predicates $S_{\sigma}$ for each $\sigma \in \omega^{<\omega}$, where $|\sigma|$ denotes the length of $\sigma$. Now if $\sigma \notin \tau$, then we will ensure that $T \models \forall \bar{x}\left(\neg S_{\sigma}(\bar{x})\right)$. Our final language will also contain an infinite sequence of $|\sigma|+$ 2-ary predicates $R_{\sigma}^{0}, R_{\sigma}^{1}, R_{\sigma}^{2}, \ldots$ for each $\sigma \in \omega^{<\omega}$. The $R_{0}^{i}$ 's will be used to ensure that there is no infinite sequence of order indiscernibles $I=\left\{i_{0}, i_{1}, \ldots\right\}$ in any decidable model $\mathcal{M}$ of $T$ such that $\mathcal{M} \models S_{\sigma}\left(i_{0}, \ldots, i_{|\sigma|}\right)$ and yet $\mathcal{M} \models$ $\neg S_{\sigma^{\wedge} 1}\left(i_{0}, \ldots, i_{|\sigma|+1}\right)$ for each $i \in \omega$, where $\sigma^{\wedge} i=\left\langle\sigma_{0}, \ldots, \sigma_{k}, i\right\rangle$ if $\sigma=\left\langle\sigma_{0}, \ldots, \sigma_{k}\right\rangle$. The idea will be to ensure that if $\mathcal{M} \models S_{\sigma}\left(i_{0}, \ldots, i_{|\sigma|}\right)$ and $\mathcal{M} \models \neg S_{\sigma \wedge}\left(i_{0}, \ldots, i_{|\sigma|+1}\right)$ for all $i \in \omega$, then the $R_{\sigma}^{i}$ 's will allow us to recursively separate a pair of recursively inseparable r.e. sets $A$ and $B$. That is, we shall ensure that if $I$ were a recursive set of order indiscernibles with the properties outlined above, then $A \subseteq\left\{j \mid \mathcal{M} \models R_{\sigma}^{j}\left(i_{0}, \ldots, i_{|\sigma|+1}\right)\right\}$ and $B \subseteq\left\{j \mid \mathcal{M} \models \neg R_{\sigma}^{j}\left(i_{0}, \ldots, i_{|\sigma|+1}\right)\right\}$ which would violate the recursive inseparability of $A$ and $B$ if $\mathcal{M}$ is decidable. It will then follow that if $I$ is an infinite set of order indiscernibles in some decidable model $\mathcal{M}$ of $T$, then for each $n>0$, there will be a $\sigma \in \tau$ with $|\sigma|=n$ such that $\mathcal{M} \models S\left(i_{0}, \ldots, i_{|\sigma|}\right)$ so that each such $I$ will code an infinite path through $T$. Finally, it will follow from the way our theory $T$ is constructed via Lemmas 1.2 and 1.3 that for every infinite path $\pi$ through $T$, there is a set of indiscernibles $I$ which codes $\pi$ as above.

We now turn to the formal definitions of the $L_{i}$ and $K_{i}$. Given $\sigma=\left\langle\sigma_{1}, \ldots, \sigma_{|\sigma|}\right\rangle$ $\in \omega^{<\omega}$, we let $\|\sigma\|=\max \left\{|\sigma|+1, \sigma_{1}, \ldots, \sigma_{|\sigma|}\right\}$. We let $m^{\leq n}=\left\{\sigma=\left\langle\sigma_{1}, \ldots, \sigma_{|\sigma|}\right\rangle \in\right.$ $\omega^{<\omega}|| \sigma \mid \leq n$ and $\sigma_{i}<m$ for each $\left.i=1, \ldots,|\sigma|\right\}$ and $m^{<n}=\left\{\sigma=\left\langle\sigma_{1}, \ldots, \sigma_{|\sigma|}\right\rangle \in\right.$ $\omega^{<\omega}|| \sigma \mid<n$ and $\sigma_{i}<m$ for each $\left.i=1, \ldots,|\sigma|\right\}$. Then for each $m \geq 1$, we let $L_{m}$ be the language whose predicates are $\left\{S_{\sigma} \mid \sigma \in m \leq m\right\} \cup\left\{R_{\tau}^{i} \mid \tau \in m^{<m}\right.$ and $\left.i<m\right\}$. Then we let $K_{m}$ be the class of all finite structures $A$ over $L_{m}$ which satisfy the following five properties.

(1) $A \models \forall x\left(S_{\phi}(x)\right)$ and for all $\sigma \in m^{\leq n}$ such that $\sigma \notin \tau, A \models \forall \bar{x}\left(\neg S_{\sigma}(\bar{x})\right)$. 
(2) For all $S_{\sigma}$ and $R_{\tau}^{i}$ in $L_{m}$ and all permutations $p$ and $p^{\prime}$,

$$
A \models \forall \bar{x}\left(S_{\sigma}(\bar{x}) \rightarrow S_{\sigma}(p(\bar{x})) \wedge \bigwedge_{i \neq j} x_{i} \neq x_{j}\right)
$$

and

$$
A \models \forall \bar{y}\left(R_{\tau}^{i}(\bar{y}) \rightarrow R_{\tau}^{i}\left(p^{\prime}(\bar{y})\right) \wedge \bigwedge_{i=j} y_{i} \neq y_{j}\right),
$$

where $\bar{x}=x_{0}, \ldots, x_{|\sigma|}$ and $p(\bar{x})$ is the permutation of $x_{0}, \ldots, x_{|\sigma|}$ induced by $p$ and $\bar{y}=y_{0}, \ldots, y_{|\tau|+1}$ and $p^{\prime}(\bar{y})$ is the permutation of $y_{0}, \ldots, y_{|\tau|+1}$ induced by $p^{\prime}$.

(3) (i) For all $\sigma \in m^{<m}$ and $i, j<m$, if $i \neq j$, then

$$
A \models \forall \bar{x}\left(S_{\sigma \wedge i}(\bar{x}) \rightarrow \neg S_{\sigma \wedge j}(\bar{x})\right) \text {. }
$$

(ii) For all $\sigma, \tau \in m^{\leq m}$ if $\sigma \subseteq \tau$, then $A \models \forall \bar{x}\left(S_{\tau}(\bar{x}) \rightarrow S_{\sigma}\left(\bar{x}_{|\sigma|}\right)\right)$, where $\bar{x}=\left(x_{0}, \ldots, x_{\tau}\right)$ and $\bar{x}_{|\sigma|}=\left(x_{0}, \ldots, x_{|\sigma|}\right)$.

(iii) For all $i \leq j<m$ and $\sigma \in m^{<m}$

$$
A \vDash \forall \bar{x}\left(S_{\sigma^{\wedge} i}(\bar{x}) \rightarrow \neg R_{\sigma}^{j}(\bar{x})\right) .
$$

(iv) For all $\sigma \in m^{<m}$ and $i<m, A \models \forall \bar{x}\left(\neg S_{\sigma}\left(\bar{x}_{|\sigma|}\right) \rightarrow \neg R_{\sigma}^{i}(\bar{x})\right)$, where $\bar{x}=$ $\left(x_{0}, \ldots, x_{|\sigma|+1}\right)$ and $\bar{x}_{|\sigma|}=\left(x_{0}, \ldots, x_{|\sigma|}\right)$.

(4) For all $j<m, k \in \omega$, and $\sigma \in m^{<m}$, if $n=\|\sigma\|<k, j \in A^{k}$, where $A^{k}\left(B^{k}\right)$ denotes the finite set of elements enumerated after $k$ steps of the effective procedure to enumerate $A(B)$, and $b=\left\langle b_{0}, \ldots, b_{k-1}\right\rangle$ is a sequence of distinct indiscernibles in $A$ with respect to the atomic formulas of $L_{n}$, then either

(a) $A \models \neg S_{\sigma}\left(b_{0}, \ldots, b_{|\sigma|}\right)$, or

(b) $A \models R_{\sigma}^{j}\left(b_{0}, \ldots, b_{|\sigma|+1}\right)$, or

(c) for some $k^{\prime} \leq k, \sigma^{\wedge} k^{\prime} \in \tau$ and it is not the case that

$$
A \vDash \neg S_{\sigma \wedge k^{\prime}}\left(b_{0}, \ldots, b_{|\sigma|+1}\right) .
$$

(We note that our definition of $\|\sigma\|$ ensures that $|\sigma|+1 \leq\|\sigma\|<k$ so that $b_{0}, \ldots, b_{|\sigma|+1}$ is an initial segment of $b_{0}, \ldots, b_{k-1}$. Also in clause (c), we are allowing that $k^{\prime} \geq m$ so that $S_{\sigma \wedge k^{\prime}}\left(b_{0}, \ldots, b_{|\sigma|+1}\right)$. However, we note that since $\bar{b} \in \mathcal{A}$, we need only consider $k \leq|\mathcal{A}|$ to verify that condition (4) holds.)

(5) For all $j<m, k \in \omega$, and $\sigma \in m^{<m}$, if $n=\|\sigma\|<k, j \in B^{k}$ and $\bar{b}=\left\langle b_{0}, \ldots, b_{k-1}\right\rangle$ is a sequence of distinct indiscernibles in $A$ with respect to the atomic formulas of $L_{n}$, then either

(i) $A \models \neg S_{\sigma}\left(b_{0}, \ldots, b_{|\sigma|}\right)$, or

(ii) $A \models \neg R_{\sigma}^{j}\left(b_{0}, \ldots, b_{|\sigma|+1}\right)$, or

(iii) for some $k^{\prime} \leq k, \sigma^{\wedge} k^{\prime} \in T$ and it is not the case that

$$
A \models \neg S_{\sigma \wedge k}\left(b_{0}, \ldots, b_{|\sigma|+1}\right) \text {. }
$$

First we must show that for each $m \geq 1, K_{m}$ is a recursive full family of structures. It is clear from properties (1)-(5) of the definition of $K_{m}$ that $K_{m}$ is closed under isomorphisms and substructures, and moreover that for any given finite structure $A$ over $L_{m}$, we can effectively decide if $A$ is in $K_{m}$. To see that $K_{m}$ has the strong amalgamation property, suppose that $A$ and $B$ are in $K_{m}$ and that 
$A|A \cap B=B| A \cap B$. We define a structure $C$ whose universe is $\operatorname{do}(A) \cup \operatorname{do}(B)$ as follows. If $\bar{c} \in A(B)$ then let $C$ interpret $S_{\sigma}$ or $R_{\tau}^{i}$ on $\bar{c}$ just as $A(B)$ does. If $\bar{c} \in C$ but $\bar{c} \notin A$ and $\bar{c} \notin B$ and $\sigma, \tau$ are of appropriate length, then

(A) $C \models \neg S_{\sigma}(\bar{c})$, and

(B) $C \models R_{\tau}^{j}(\bar{c})$ iff $\bar{c}$ is a sequence of distinct elements, $j \in A^{p}$, where $p=\operatorname{card}(C)$, and $C \models S_{\tau}\left(c_{|\tau|}\right)$, where $c_{|\tau|}$ is any subsequence of $\bar{c}$ of length $|\tau|+1$.

To see that $C \in K_{m}$, we must check properties (1)-(5). Properties (1) and (2) are immediate, properties (3)(i)-(iii) follow because $C \models S_{\sigma}(\bar{c})$ iff $\bar{c} \in A$ or $\bar{c} \in B$, and property (3)(iv) follows from clause (B) of our definition of $C$. For properties (4) and (5), suppose that $j<m, k \in N, \sigma \in(m-1)^{\leq m-1}, n=\|\sigma\|<k$, and $\bar{b}=\left\langle b_{0}, \ldots, b_{k-1}\right\rangle$ is a sequence of distinct indiscernibles in $C$ with respect to the atomic formulas of $L_{n}$. Now if $\bar{b} \subseteq A$ or $\bar{b} \subseteq B$, then properties (4) and (5) hold for $\bar{b}$ since $A, B \in K_{m}$. Thus assume $\bar{b} \nsubseteq A$ and $\bar{b} \nsubseteq B$. Now if $\sigma \neq \phi$, then since $\bar{b}$ is a sequence of indiscernibles, our definitions force $C \models \neg S_{\sigma}\left(b_{0}, \ldots, b_{|\sigma|-1}\right)$ so that clause (a) of conditions (4) and (5) hold. Finally if $\sigma=\phi$, then $j \in A^{k}$ implies that $R_{\phi}^{j}\left(b_{0}, b_{1}\right)$ holds in $C$ and $j \in B^{k}$ implies $\neg R_{\phi}^{j}\left(b_{0}, b_{1}\right)$ holds in $C$ by clause (B) of our definition of $C$ so that (4) and (5) hold for all $\bar{b}$ and $\sigma$. Thus $C$ amalgamates $A$ and $B$ in $K_{m}$.

Next, in order to apply 1.3 we must show that for all $m<n, A \in K_{m}$, and $B^{*} \in K_{n}$ (b) $B^{*} \mid L_{m} \in K_{m}$ and (c) if $B^{*} \mid L_{m}$ is a submodel of $A$, then $A$ can be expanded to $A^{*} \in K_{m}$ such that $B^{*}$ is a submodel of $A^{*}$. It is easy to see that $B^{*} \mid L_{m}$ satisfies conditions (1)-(3) of the definition of $K_{m}$. The fact that $B^{*} \mid L_{m}$ satisfies conditions (4) and (5) of the definition of $K_{m}$ follows from the fact that in conditions (4) and (5) we referred to sets of indiscernibles with respect to $L_{m}$. Now consider (c). By induction we may assume that $n=m+1$. We define $A^{*}$ as follows. Fix $\bar{a}=\left(a_{0}, \ldots, a_{k}\right)$ in $A$ and $S_{\sigma^{\wedge} j}$ and $R_{\sigma}^{j}$ in $L_{n}-L_{m}$ with $\sigma$ of length $k-1$. If $\bar{a} \in B^{*}$, then we are forced to define $A^{*} \models S_{\sigma{ }^{\wedge} j}(\bar{a})$ iff $B^{*} \models S_{\sigma}{ }^{\wedge}(\bar{a})$ and $A^{*} \models R_{\sigma}^{j}(\bar{a})$ iff $B^{*} \models R_{\sigma}^{j}(\bar{a})$. Now suppose that for some $l, a_{l} \in B^{*}$. Our strategy is to allow $A^{*} \models S_{\sigma^{\wedge} j}(\bar{a})$ only in those cases in which $A$ meets conditions (4) or (5) by not $A \models \neg S_{\sigma^{\wedge} j}(\bar{a})$ and $\sigma^{\wedge} j \in \tau$. To meet (4) and (5) in the other cases we shall consider a listing of $A^{p}$, where $p=\operatorname{card}(\mathcal{A})$, to determine whether $A^{*} \models R_{\sigma}^{j}$. So let $\mathcal{A}^{*} \models S_{\sigma^{\wedge} j}(\bar{a})$ iff $\sigma \in m^{<m}, j=m$, and

(A1) $\sigma^{\wedge} m \in \mathcal{T}$

(A2) the elements of $\bar{a}$ are distinct;

(A3)(i) $A \models \neg S_{\sigma} \wedge i(\bar{a})$ for all $i<m$; and

(ii) $A \models S_{\sigma}(\bar{c})$ for all subsequences $\bar{c}$ of $\bar{a}$ of length $k$.

Let $A^{*} \models R_{\sigma}^{j}(\bar{a})$ iff

(B2) the elements of $\bar{a}$ are distinct;

(B3)(iii) $A^{*} \models \neg S_{\sigma^{\wedge} i}(\bar{a})$ for all $i<j$;

(iv) $A^{*} \models S_{\sigma}(\bar{c})$ for all subsequences $\bar{c}$ of $\bar{a}$ of length $k$;

(B4) $j \in A^{p}$, where $p=\operatorname{card}(\mathcal{A})$.

It is easy to check that $A^{*}$ satisfies conditions (1)-(3) of the definition of $K_{n}$. (The clauses of the above definitions are labeled to correspond to the conditions on $K_{n}$ and elements of $B^{*}$ behave properly because $B^{*} \in K_{n}$.) For conditions (4) and (5) suppose that $j \leq m, \sigma \in(m+1)^{<m+1}, n^{\prime}=\|\sigma\|<K$ and that $\bar{b}=\left(b_{0}, \ldots, b_{k}\right)$ is a sequence of distinct indiscernibles with respect to the atomic formulas of $L_{n^{\prime}}$. Since $B^{*} \in K_{n}$ we may assume that some $b_{l} \notin B^{*}$ and by condition (2) we may 
assume that $l=0$. First suppose that $n^{\prime}<m$ and that $A^{*} \models S_{\sigma}\left(b_{0}, \ldots, b_{|\sigma|}\right)$. Thus $\sigma \in m^{<m}$ and $A \models S_{\sigma}\left(b_{0}, \ldots, b_{|\sigma|}\right)$. Since $A \in K_{m}, A$ satisfies either (b) or (c) of conditions (4) and (5). If $A$ satisfies (b) of either (4) or (5) then so does $A^{*}$. Suppose that $A$ satisfies (c) of either (4) or (5) and that $k^{\prime}$ is the smallest integer such that not $A \models \neg S_{\sigma \wedge} k^{\prime}\left(b_{0}, \ldots, b_{|\sigma|+1}\right)$ and $\sigma^{\wedge} k^{\prime} \in \tau$. If $k^{\prime}<m$ or $k^{\prime}>m$ then $A^{*}$ satisfies (c) by default. If $k^{\prime}=m$, then (A1) and (A3)(i) hold for $\bar{a}=\left(b_{0}, \ldots, b_{|\sigma|+1}\right)$ by the choice of $k^{\prime}$. Since $A \models S_{\sigma}\left(b_{0}, \ldots, b_{|\sigma|}\right)$ and $\bar{a}$ is a sequence of indiscernibles, it is easy to see that conditions (A2) and (A3)(ii) hold. Thus our definition of $A^{*}$ forces $A^{*} \models S_{\sigma^{\wedge} m}(\bar{a})$; so (4)(c) and (5)(c) hold.

Finally, suppose that $n^{\prime}=m$ and $A^{*} \models S_{\sigma}\left(b_{0}, \ldots, b_{|\sigma|}\right)$. Again the fact that $\bar{a}=\left(b_{0}, \ldots, b_{|\sigma|+1}\right)$ is a sequence of indiscernibles ensures that (B2) and (B3)(iv) hold for $\bar{a}$. Our construction guarantees that (B3)(iii) holds for $\bar{a}$. Thus by condition (B4) of our definition of $\mathcal{A}^{*}, \mathcal{A}^{*} \models R_{\sigma}^{j}(\bar{a})$ iff $j \in A^{p}$, where $p=\operatorname{card}(\mathcal{A})$; so (4B) and $(5 \mathrm{~b})$ are met.

We can now apply Lemma 1.3 to construct an $L(Q)$-decidable theory $T$ which admits elimination of quantifiers, where $L=\bigcup_{i=1}^{\infty} L_{i}, T=\bigcup_{i=1}^{\infty} T_{i}$, and $T_{i}$ is the $\omega$-categorical theory constructed from $K_{i}$ via Lemma 1.2. Now suppose that $\mathcal{M}$ is any decidable model $T$ and $I=\left\langle i_{0}, i_{1}, \ldots,\right\rangle$ is an infinite sequence of order indiscernibles in $\mathcal{M}$. We claim that there is a unique path $\pi=\left(\pi_{0}, \pi_{1}, \pi_{2}, \ldots\right)$ through $\tau$ such that for all $n, \mathcal{M} \models S_{\pi_{n}}\left(i_{0}, \ldots, i_{n}\right)$. The uniqueness of $\pi$ follows immediately from condition (3)(i) of our definition of $K_{m}$. To see that $\pi$ exists, note that $\pi_{0}=\phi$ since by condition (1) of our definition of $K_{m}, \mathcal{M} \models S_{\phi}\left(i_{0}\right)$. Now if $\mathcal{M} \models S_{\pi_{n}}\left(i_{0}, \ldots, i_{n}\right)$, then it is impossible that $\mathcal{M} \models \neg S_{\pi_{n} i}\left(i_{0}, \ldots, i_{n+1}\right)$ for all $i \in \omega$. That is, if $\mathcal{M} \models \neg S_{\pi_{n} i}\left(i_{0}, \ldots, i_{n+1}\right)$ for all $i$, then by conditions (4) and (5) of our definition of $K_{m}, A \subseteq R=\left\{j \mid \mathcal{M} \models R_{\pi_{n}}^{i}\left(i_{0}, \ldots, i_{n+1}\right)\right\}$ and $B \subseteq \bar{R}=\left\{j \mid \mathcal{M} \models \neg R_{\pi_{n}}^{i}\left(i_{0}, \ldots, i_{n+1}\right)\right\}$. Since $\mathcal{M}$ is decidable, $R$ and $\bar{R}$ are recursive sets which violate the fact that $A$ and $B$ are recursively inseparable. Thus there exists a unique $j$ such that $\pi_{n+1}=\pi_{n} \wedge j$ and $\mathcal{M} \vDash S_{\pi_{n+1}}\left(i_{0}, \ldots, i_{n+1}\right)$. Clearly $\pi$ is recursive in $I$ by the decidability of $\mathcal{M}$. Thus there is a partial recursive function $\chi_{e}$ such that if $I=\left(i_{0}, i_{1}, \ldots\right)$ is an infinite sequence of order indiscernibles in $\mathcal{M}$, then $\chi_{e}^{I}=\pi_{I}=\left\langle\pi_{0}, \pi_{1}, \ldots\right\rangle$, where $\forall n\left(\mathcal{M} \models S_{\pi_{n}}\left(i_{0}, \ldots, i_{n}\right)\right)$. Thus the correspondence $I \rightarrow \pi_{I}$ provides us with one of the two correspondences required to prove $P(\tau) \approx I(\mathcal{M})$.

To complete the proof that $P(\tau) \approx I(\mathcal{M})$, we must show that given any path $\pi=\left(\pi_{0}, \pi_{1}, \ldots\right)$ in $P(\tau)$, we can construct a sequence of order indiscernibles $I_{\pi}=$ $\left\langle i_{0}, i_{1}, \ldots\right\rangle$ of $\mathcal{M}$ such that $I_{\pi}$ is recursive in $\pi$ and $\pi_{I_{\pi}}=I_{\pi}$. Now for each $n \geq 0$, consider the formula $\rho_{n}$, where

$$
\begin{aligned}
\rho_{n}\left(x_{0}, \ldots, x_{n}\right)=\bigwedge_{r \leq n} & {\left[\bigwedge_{0 \leq i_{0}<\cdots<i_{r} \leq m} S_{\pi_{r}}\left(x_{i_{0}}, \ldots, x_{i_{r}}\right)\right.} \\
& \left.\wedge \bigwedge_{j \in A_{r}} R_{\pi_{r-1}}^{j}\left(x_{i_{0}}, \ldots, x_{i_{r}}\right) \wedge \bigwedge_{j \in B_{r}} \neg R_{\pi_{r-1}}^{j}\left(x_{i_{0}}, \ldots, x_{i_{r}}\right)\right],
\end{aligned}
$$

$m=\left\|\pi_{r}\right\|, \pi_{r}=\pi_{r-1} k, A_{r}=\left\{j<k \mid j \in A^{\left\|\pi_{r}\right\|}\right\}$, and $B_{r}=\left\{j<m \mid j \notin A_{r}\right\}$. It is easy to see from our definition of $K_{m}$ that $\rho_{n}$ is consistent with $T_{m}$ and hence with $T$. Moreover, it follows from conditions (1)-(3) of our definitions of 
the $K_{m}$ 's that $\rho_{n}\left(x_{0}, \ldots, x_{n}\right)$ implies whether or not each $S_{\sigma}\left(x_{i_{0}}, \ldots, x_{i_{|\sigma|}}\right)$ and $R_{\tau}^{j}\left(x_{i_{0}}, \ldots, x_{i_{|\tau|+1}}\right)$ holds for all $|\sigma| \leq n,|\tau| \leq n-1$, and $i_{j} \leq n$ so that by the elimination of quantifiers of $T, \rho_{n}$ is complete with respect to $T$. Note also that $T \models \forall \bar{x}^{\wedge} y\left(\rho_{n+1}\left(\bar{x}^{\wedge} y\right) \rightarrow \rho_{n}(\bar{x})\right)$. It follows that if $\mathcal{N}$ is model $T$ and $I=\left\langle i_{0}, i_{1}, \ldots\right\rangle$ is a sequence of elements of $\mathcal{N}$ such that $\mathcal{N} \models \rho_{n}\left(i_{0}, \ldots, i_{n}\right)$ for all $n$, then $I$ is a sequence of indiscernibles in $\mathcal{N}$. Now in our given decidable model $\mathcal{M}$ of $T$, we define the sequence $I_{\pi}=\left\langle i_{0}, i_{1}, \ldots\right\rangle$ as follows:

(a) $i_{0}=\mu a\left(a \in \mathcal{M}\right.$ and $\left.\mathcal{M} \models S_{\phi}(a)\right)$.

(b) Having defined $i_{0}, \ldots, i_{n}$ so that for each $k \leq n, \mathcal{M} \vDash \rho_{k}\left(i_{0}, \ldots, i_{k}\right)$, let $i_{n+1}=\mu a\left(a \in \mathcal{M}, a>n\right.$, and $\left.\mathcal{M} \models \rho_{n+1}\left(i_{0}, \ldots, i_{n}, a\right)\right)$.

To see that $i_{n+1}$ exists, let $m=\left\|\tau_{n+1}\right\|$ and observe that $T_{m}=T \nmid L_{m}$. If we consider $\mathcal{M} \mid L_{m}$ as a model of the $\omega$-categorical theory $T_{m}$, then by Lemma 1.2 , $B=\mathcal{M} \mid\left\{i_{0}, \ldots, i_{n}\right\}$ must be a finite structure in $K_{m}$. Moreover there is clearly a finite structure $A=\left\{a_{0}, \ldots, a_{n+1}\right\}$ in $K_{m}$ so that $A \models \rho_{n+1}\left(a_{0}, \ldots, a_{n+1}\right)$. Now using the facts that $K_{m}$ is closed under isomorphisms and has the strong amalgamation property, one can easily show that Lemma 1.2 implies that $T_{m} \vDash$ $\forall \bar{x}\left(\rho_{n}(\bar{x}) \rightarrow \exists y_{0}, \ldots, y_{n+1}\left(\bigwedge_{i \neq j} y_{i} \neq y_{j} \wedge \rho_{n+1}\left(\bar{x}, y_{i}\right)\right)\right)$. Thus $T$ also satisfies the same sentence so that $i_{n+1}$ is well defined for all $n$. Note that $I_{\pi}$ is clearly recursive in the sequence of formulas $\left\langle\rho_{1}, \rho_{2}, \ldots\right\rangle$ which in turn is recursive in $\pi$. Moreover, it is easy to see that $\pi_{I_{\pi}}=\pi$. Thus there is a partial recursive function $\chi_{f}$ such that $\chi_{f}^{\pi}=I_{\pi}$ for all $\pi \in P(\tau)$ so that $P(\tau) \approx I(\mathcal{M})$ for all decidable models $\mathcal{M}$ of $T$.

We note that if $T$ is the theory constructed in the proof of Theorem 2.4 and $T^{\prime}$ equals $T$ restricted to the language $\mathcal{L}=\left\{S_{\sigma} \mid \sigma \in \omega^{<\omega}\right\}$, then $T^{\prime}$ is also a decidable theory which admits the elimination of quantifiers. Since the omitting types theorem [1] is effective, it follows that $T^{\prime}$ has a decidable model $\mathcal{M}^{\prime}$ which omits all the types

$$
\begin{aligned}
\rho_{\sigma}\left(x_{0}, \ldots, x_{|\sigma|+1}\right)= & \left\{S_{\sigma}\left(x_{i_{0}}, \ldots, x_{i_{|\sigma|}}\right)\left|0 \leq i_{0}<\cdots<i_{|\sigma|} \leq\right| \sigma \mid+1\right\} \\
& \cup\left\{\neg S_{\sigma^{\wedge} i}\left(x_{0}, \ldots, x_{|\sigma|+1}\right) \mid i \in \omega\right\} .
\end{aligned}
$$

It then follows by essentially the same analysis as in Theorem 2.4 that there is an effective $1: 1$ correspondence between $\omega$-types of infinite sets of order indiscernibles which are realized in $\mathcal{M}^{\prime}$ and paths through $\tau$. Moreover we note that our construction ensures that every sequence $I=\left\langle i_{R}: R<\omega\right\rangle$ of order indiscernibles of $\mathcal{M}^{\prime}$ is, in fact, a set of total indiscernibles in $\mathcal{M}^{\prime}$, i.e. for every $1: 1$ map $\sigma:(0, \ldots, n-1) \rightarrow \omega$ and every sentence $\rho, \mathcal{M} \models \rho\left(i_{0}, \ldots, i_{n-1}\right)$ iff $\mathcal{M} \models \rho\left(i_{\sigma(0)}, \ldots, i_{\sigma(n-1)}\right)$. Thus we have the following.

COROLLARY 2.5. For any recursive $\omega$-branching tree $\tau$, there exists a decidable model $\mathcal{M}^{\prime}$ such that there is an effective $1: 1$ correspondence between $\omega$-types of infinite sets of indiscernibles realized in $\mathcal{M}$ and infinite paths through $\tau$.

We can use the correspondence proved in Theorem 2.4 to prove a number of interesting corollaries by simply considering recursive $\omega$-branching trees with various special properties. For example, if we let $\tau$ be a recursive $\omega$-branching tree with no infinite paths through $\tau$, we get the following corollary first proved in [6].

COROLlaRY 2.6. There exists a decidable $L(Q)$-decidable theory $T$ such that no decidable model $\mathcal{M}$ of $T$ has an infinite set of order indiscernibles. 
In $[8]$, it is proved that there exist recursive $\omega$-branching trees $\tau^{\prime}$ and $\left\{\tau_{n}: n<\right.$ $\omega\}$ such that there are infinite branches through $\tau^{\prime}$ but there are no infinite branches through $\tau^{\prime}$ which are hyperarithmetic and for each $n, \tau_{n}$ has exactly one infinite branch through $\tau_{n}$ which is Turing equivalent to $H(n)$. (Here $H(0)=\phi$, $H\left(2^{y}\right)=H(y)^{\prime}$, the jump of $H(y)$, and $\left.H\left(3 \cdot 5^{e}\right)=\left\{(x, y) \mid x \in H\left(\rho_{e}(y)\right)\right\}.\right)$ Thus from the correspondence in Theorem 2.4, we can derive the following

COROLLARY 2.7. (i) There exist an $L(Q)$-decidable theory $T^{\prime}$ such that every decidable model $\mathcal{M}^{\prime}$ of $T^{\prime}$ has an infinite set of total indiscernibles, but there is no decidable model $\mathcal{M}^{\prime}$ of $\tau^{\prime}$ which has an infinite set of order indiscernibles which is hyperarithmetic.

(ii) For each $n \in \omega$, there exists a decidable model $\mathcal{M}_{n}^{\prime}$ such that there is only one $\omega$-type $\theta_{n}$ of an infinite set of indiscernibles which is realized in $\mathcal{M}_{n}^{\prime}$ and $\operatorname{deg} \theta_{n}=$ $\operatorname{deg} H(n)$.

Finally the correspondence of Theorem 2.4 allows us to classify the complexity of the predicate " $M$ is a decidable model with an infinite set of order indiscernibles." That is, let $L=\bigcup_{i=1}^{\infty} L_{i}$ be the language of Theorem 2.5. Then we say that $e=\#\langle k, l\rangle$ is a Gödel number of a decidable model $\mathcal{M}$ of $T$ if $\chi_{k}$ is the characteristic function of $\operatorname{Do}(\mathcal{M})$ and $\chi_{l}$ is the characteristic function of the satisfaction predicate of $\mathcal{M}$. It is easy to see that $\mathcal{U}=\{e: e$ is a Gödel number of a decidable model $\mathcal{M}$ over $L$ which contains an infinite set of indiscernibles $\}$ is a $\Sigma_{1}^{1}$-predicate since $e \in \mathcal{U}$ iff $\exists f(\langle f(i): i \in \omega\rangle$ is an infinite set of order indiscernibles in $\mathcal{M}$ and $\mathcal{M}$ is a decidable model over $L)$ and the predicate " $\langle f(i): i \in \omega\rangle$ is an infinite set of order indiscernibles in $\mathcal{M}$ and $\mathcal{M}$ is a decidable model over $L$ " is easily seen to be arithmetic. We say that $e$ is a Gödel number of a recursive $\omega$-branching tree $\tau$ if $\chi_{e}$ is the characteristic function of $\tau$. In [8], it is shown that $\mathcal{Y}=\{e: e$ is a Gödel number of a recursive $\omega$-branching tree $\tau$ with at least one infinite branch through $\tau\}$ is a complete $\Sigma_{1}^{1}$-predicate. The correspondence in Theorem 2.4 and the fact that the Henkin construction of a model from a consistent theory $T$ is uniform and effective shows that $\mathcal{Y}$ is $1: 1$ reducible to $\mathcal{U}$. Thus we have the following

COROLLARY 2.8. \{e:e is a Gödel number of decidable model $\mathcal{M}$ over $L$ which contains an infinite set of order indiscernibles $\}$ is a complete $\Sigma_{1}^{1}$-set.

3. Indiscernibles in decidable $\omega$-categorical models. In this section, we shall explore the possible degrees of infinite sets of order indiscernibles in decidable $\omega$-categorical models. First, we shall show that the problem of finding an infinite set of order indiscernibles in a decidable model $\mathcal{M}$ of an $\omega$-categorical theory $T$ with decidable atoms is essentially equivalent to the problem of finding an infinite path through an infinite recursive binary tree. Note that since Ehrenfeucht and Mostowski proved that every countable first order theory $T$ which has an infinite model has a countable model with an infinite set of order indiscernibles, every $\omega$ categorical model $\mathcal{M}$ has an infinite set order indiscernibles. Hence, if $P(\tau) \approx I(\mathcal{M})$ for a decidable $\omega$-categorical model $\mathcal{M}$, then $P(\tau) \neq \varnothing$, i.e., there is at least one infinite path through $\tau$. Thus for the rest of this section we shall consider only the class of infinite binary trees which by König's lemma is the class of binary trees $\tau$ with at least one infinite path. 
First, we shall prove the analogue of Theorem 2.4 for infinite recursive binary trees and decidable $\omega$-categorical models. We remark that all decidable models of a decidable $\omega$-categorical theory with decidable atoms are recursively isomorphic.

THEOREM 3.1. For any infinite recursive binary tree $\tau$, there exists a decidable $\omega$-categorical theory $T$ with decidable atoms such that for any decidable model $\mathcal{M}$ of $T, P(\tau)=I(\mathcal{M})$.

PROOF. As in the proof of Theorem 2.4, we shall use Lemma 1.3 to construct our theory $T$. Thus we must specify an increasing sequence of languages, $L_{1} \subseteq$ $L_{2} \subseteq L_{3} \subseteq \ldots$, with only relation symbols and an effective sequence $K_{1}, K_{2}, \ldots$ of classes of finite structures satisfying conditions (a)-(c) of Lemma 1.3 so that if $T_{i}$ is the $\omega$-categorical $L_{i}(Q)$-decidable theory constructed from $K_{i}$ via Lemma 1.2, then the theory $T=\bigcup_{i=1}^{\infty} T_{i}$ is the desired theory.

Fix an arbitrary infinite recursive binary tree $\tau$. For each $i \geq 1, L_{i}$ will consist of the predicates $S_{1}, \ldots, S_{i}$ plus equality, where $S_{j}$ is a $(j+1)$ ary predicate for each $j$. We shall ensure that our final theory $T$ contains the following sentences.

(1) For all $n$ and permutations $\sigma$ of $\{0, \ldots, n\}$

$$
\forall x_{0} \cdots \forall x_{n}\left(S_{n}\left(x_{0}, \ldots, x_{n}\right) \rightarrow S_{n}\left(x_{\sigma(0)}, \ldots, x_{\sigma(n)}\right) \wedge \bigwedge_{0 \leq j<l \leq n} x_{j} \neq x_{l}\right) .
$$

Note that the sentences in (1) and the fact that $T$ admits the elimination of quantifiers will ensure that for each $n$, there will be only finitely many $n$-types so that $T$ will be $\omega$-categorical. Also, it is clear by the elimination of quantifiers that $T$ will have decidable atoms.

We can identify $n$-types of indiscernibles in $T$ with finite sequences of 0 's and 1's. That is, if $I=\left\langle i_{n}: n \in \omega\right\rangle$ is a set of order indiscernibles in some model $\mathcal{M}$ of $T$, then we shall say the $n$-type of $I$ corresponds to the sequence $\left\langle\tau_{1}, \ldots, \tau_{n}\right\rangle$ and write $\tau(I)=\left\langle\tau_{1}, \ldots, \tau_{n}\right\rangle$ if for each $j \leq n, \tau_{j}=1$ if $\mathcal{M} \models S_{j}\left(i_{0}, \ldots, i_{j}\right)$ and $\tau_{j}=0$ otherwise. In exactly the same way, we can identify atomic $n$-types, i.e. types restricted to atomic and negations of atomic formulas, of indiscernibles over $L_{n}$ with finite sequences of 0 's and 1's. This given, we can now describe the required full families of finite structures. For each $n$, let $K_{n}$ consist of all finite structures $A$ over $L_{n}$ such that:

(a) For all $j \leq n$ and permutations $\sigma$ of $\{\sigma, \ldots, j\}$

$$
A \models \forall x_{0} \cdots \forall x_{j}\left(S_{j}\left(x_{0}, \ldots, x_{j}\right) \rightarrow S_{j}\left(x_{\sigma(0)}, \ldots, x_{\sigma(j)}\right) \wedge \bigwedge_{0 \leq l<k \leq j} x_{l} \neq x_{k}\right) .
$$

(b) For each $1 \leq j \leq n$ and sequence of 0 's and 1's $\left\langle\tau_{1}, \ldots, \tau_{j}\right\rangle$, there exists a set of distinct order indiscernibles $\left\langle b_{1}, \ldots, b_{s}\right\rangle$ with respect to the atomic formulas of $L_{j}$ in $A$ which have $j$-type $\left\langle\tau_{1}, \ldots, \tau_{j}\right\rangle$, only if there is a node $\left\langle a_{1}, \ldots, a_{s}\right\rangle \in \tau$ at level $s$ such that $\left\langle a_{1}, \ldots, a_{s}\right\rangle$ extends $\left\langle\tau_{1}, \ldots, \tau_{j}\right\rangle$, where $\tau_{i}=1$ if $A \models S_{i}\left(b_{0}, \ldots, b_{i}\right)$ and $\tau_{i}=0$ otherwise.

First we must check that the families of structures $K_{1}, K_{2}, \ldots$ satisfy conditions (a)-(c) of Lemma 1.3. For fixed $n$, it is easy to see by our definitions that $K_{n}$ is closed under isomorphisms and substructures and we can effectively decide if a finite structure $\mathcal{A}$ over $L_{n}$ is in $K_{n}$. Thus to show that $K_{n}$ is a recursive full family 
of structures we need only show that $K_{n}$ has the strong amalgamation property. So assume that $A$ and $B$ are finite structures in $K_{n}$ and that $A|A \cap B=B| A \cap B$. Since $\tau$ is infinite, there is an infinite path $\pi=\left\langle\pi_{1}, \pi_{2}, \ldots\right\rangle$ through $\tau$. Fix $\pi$ and then let $C$ be the finite structure with domain equal to $\operatorname{Do}(A) \cup \operatorname{Do}(B)$ such that for all $j \leq n$ and $c_{0}, \ldots, c_{j} \in C$

(*) $A \models S_{j}\left(c_{0}, \ldots, c_{j}\right)$ iff either

(i) $c_{0}, \ldots, c_{j} \in A$ and $A \models S_{j}\left(c_{0}, \ldots, c_{j}\right)$, or

(ii) $c_{0}, \ldots, c_{j} \in B$ and $B \models S_{j}\left(c_{0}, \ldots, c_{j}\right)$, or

(iii) not (i) and (ii), $c_{0}, \ldots, c_{j}$ are distinct, and $\pi_{j}=1$.

It is easy to check that $C$ satisfies conditions (a) and (b) of the definition of $K_{n}$ since if $b_{1}, \ldots, b_{s}$ is a set of distinct order indiscernibles in $C$ with respect to the atomic formulas of $L_{j}$ for some $j \leq n$ and $\left\langle b_{1}, \ldots, b_{s}\right\rangle$ is not contained entirely within $A$ or entirely in $B$, then condition (*) ensures that $\left\langle\tau\left(b_{1}, \ldots, b_{m}\right)\right\rangle=$ $\left\langle\pi_{1}, \ldots, \pi_{j}\right\rangle$. Since $\left\langle\pi_{1}, \ldots, \pi_{j}\right\rangle$ is a node on $\pi$, then $\left\langle\pi_{1}, \ldots, \pi_{s}\right\rangle$ is a node in $\tau$ at level $s$ extending $\left\langle\pi_{1}, \ldots, \pi_{j}\right\rangle$. Thus $C$ amalgamates $A$ and $B$ in $K_{n}$.

For conditions (b) and (c) of Lemma 1.3, assume that $m<n, A \in K_{m}$ and $B^{*} \in K_{n}$. It is easy to see that by the form of our definitions of $K_{m}$ and $K_{n}$ that $B=B^{*} \mid K_{n} \in K_{m}$. Finally we must show that if $B$ is a submodel of $A$ then $A$ can be expanded to $A^{*} \in K_{n}$ such that $B^{*}$ is a submodel of $A^{*}$. Clearly by induction we can assume that $n=m+1$. To define $A^{*}$ we need only specify which $(n+1)$-tuples $\bar{a}=\left(a_{0}, \ldots, a_{n}\right)$ satisfy $S_{n}$. If $\bar{a} \in B^{*}$, let $A^{*} \models S_{n}(\bar{a})$ iff $B^{*} \models S_{n}(\bar{a})$. Now suppose that for some $l, a_{l} \notin B^{*}$. Let $A^{*} \models \neg S_{n}(\bar{a})$ if $\bar{a}$ is not a set of distinct indiscernibles in $A$ with respect to the atomic formulas of $L_{m}$. Otherwise, find the maximum $s \geq n+1$ such that there exist $b_{n+1}, \ldots, b_{s} \in \mathcal{A}$ such that $\left\langle a_{0}, \ldots, a_{n}, b_{n+1}, \ldots, b_{s}\right\rangle$ is a set of order indiscernibles in $A$ with respect to the atomic formulas of $L_{m}$. Since $A \in K_{m}$, we know that there exists a node $\left\langle\tau_{1}, \ldots, \tau_{s}\right\rangle$ in $\tau$ at level $s$, where $\left\langle\tau_{1}, \ldots, \tau_{s}\right\rangle$ is a sequences of 0 's and 1's corresponding to the indiscernibles $\left\langle a_{0}, \ldots, a_{n}, b_{n+1}, \ldots, b_{s}\right\rangle$. Assume $\left\langle\tau_{1}, \ldots, \tau_{s}\right\rangle$ is the lexicographically least node in $\tau$ with the property above and let $A^{*}=S_{n}\left(a_{0}, \ldots, a_{n}\right)$ iff $\tau_{n}=1$. Clearly $B^{*}$ is a submodel of $A^{*}$ and $A^{*}$ satisfies condition (1) of the definition of $K_{n}$. To see that $A^{*}$ satisfies condition (2) of the definition of $K_{n+1}$ we need only check that if $\left\langle b_{1}, \ldots, b_{s}\right\rangle$ is a set of distinct order indiscernibles in $A^{*}$ with respect to the atomic formulas $L_{n}$ and $\tau\left(\left\langle b_{1}, \ldots, b_{s}\right\rangle\right)=\left\langle\tau_{1}, \ldots, \tau_{n+1}\right\rangle$, there is a node at level $s$ in $\tau$ of the form $\left\langle\tau_{1}, \ldots, \tau_{n+1}, \tau_{n+2}, \ldots, \tau_{s}\right\rangle$. If $\left\langle b_{1}, \ldots, b_{s}\right\rangle$ is contained in $B^{*}$ this follows from $B^{*} \in K_{n}$. Otherwise it follows easily from our definition of $\AA^{*}$. Thus $\AA^{*} \in K_{n}$ and we can apply Lemma 1.2 .

So let $T$ be the $L(Q)$-decidable theory constructed from $K_{1}, K_{2}, \ldots$ via Lemma 1.3. Because $T \nmid L_{n}=T_{n}$ for all $n$, where $T_{n}$ is the theory constructed from $K_{n}$ by Lemma 1.2 , it is easy to see that the sentences of $(1)$ are satisfied by $T$. Thus by our remarks preceding the definitions of the $K_{n}$ 's, $T$ is a $\omega$-categorical theory with decidable atoms. From condition (b) of our definitions of the $K_{n}$ 's, it is straightforward to prove that if $I=\left\langle i_{n}: n \in \omega\right\rangle$ is a set of order indiscernibles in a model of $T$ and $\tau(I)=\left\langle a_{1}, a_{2}, \ldots\right\rangle$, then $\pi=\left\langle a_{1}, a_{2}, \ldots\right\rangle$ is an infinite path through $\tau$. Note also that by the sentences in (a) and the elimination of quantifiers for $T$, every set of order indiscernible is, in fact, a set of total indiscernibles. Now fix any decidable model $\mathcal{M}$ of $T$. Since $\mathcal{M}$ is decidable, it is clear that the correspondence $I \rightarrow \tau(I)=\pi_{1}$ is effective so that there is an $f$ such that $\chi_{f}^{I}=\pi_{I}$ for all infinite 
sets of order indiscernibles in $I$. To complete the proof of the theorem, we must show that there is an effective correspondence $\pi \rightarrow I_{\pi}$ between paths $\pi \in P(\tau)$ and indiscernibles $I_{\pi} \in I(\mathcal{M})$, where $\tau\left(I_{\pi}\right)=\pi$. Note that for each node $\mathfrak{n} \in \tau$ of length $n>0$ in $\tau$, there is a sequence $\rho_{\mathfrak{n}}\left(x_{1}, \ldots, x_{n}\right)$ in $L_{n}$ which says " $\left\langle x_{1}, \ldots, x_{n}\right\rangle$ is a set distinct order indiscernibles with respect to the atomic sentences in $L_{n}$ such that $\tau\left(\left\langle x_{1}, \ldots, x_{n}\right\rangle\right)=\mathfrak{n}$." By the elimination of quantifiers for $T$ and the sentences in (a), it follows that $\rho_{\mathrm{n}}$ is an atom in the Lindenbaum algebra $B_{n}(T)$. Moreover, if $\mathfrak{n}$ is a node on an infinite path through $\tau$, it is easy to see that $\rho_{\mathrm{n}}$ must be realized in $T$. That is, $T \nmid L_{n}=T_{n}$ and $T_{n}$ contains the sentence $\exists x_{1} \ldots \exists x_{n} \rho_{n}\left(x_{1}, \ldots, x_{n}\right)$ since in $K_{n}$ there are structures $\mathcal{A}$ with arbitrarily large sets of indiscernibles $I$ with $\tau(I)=\mathfrak{n}$. Then given an infinite path $\pi=\left\langle\tau_{1}, \tau_{2}, \ldots\right\rangle$ through $\tau$, let $\mathfrak{n}_{j}=\left\langle\tau_{1}, \ldots, \tau_{j}\right\rangle$ for $j=1,2, \ldots$ Define a sequence $a_{1}, a_{2}, \ldots$ in $\mathcal{M}$ as follows:

(i) $a_{1}=\mu x\left(x \in \mathcal{M} \& \mathcal{M} \models \rho_{\mathfrak{n}_{1}}\left(a_{1}\right)\right)$.

(ii) Having defined $a_{1}, \ldots, a_{k}$, let

$$
a_{k+1}=\mu x\left(x \in \mathcal{M} \& \mathcal{M} \models \rho_{\mathfrak{n}_{k+1}}\left(a_{1}, \ldots, a_{k}, x_{k+1}\right)\right) .
$$

Note $\mathcal{M} \models \exists x_{1} \ldots \exists x_{k+1} \rho_{n_{k+1}}\left(x_{1}, \ldots, x_{k+1}\right)$ so that there exists $b_{1}, \ldots, b_{k+1}$ in $\mathcal{M}$ such that $\mathcal{M} \vDash \rho_{\mathfrak{n}_{k+1}}\left(b_{1}, \ldots, b_{k+1}\right)$. But then $b_{1}, \ldots, b_{k}$ and $a_{1}, \ldots, a_{k}$ both satisfy the same $n$-type since they both satisfy the atom $\rho_{\mathfrak{n}_{k}}$. Since $\mathcal{M}$ is $\omega$-categorical, $\mathcal{M}$ is homogeneous so that $a_{k+1}$ must exist for all $k$. It follows that if $I_{\pi}=\left\langle a_{1}, a_{2}, \ldots\right\rangle$, then it is a set of indiscernibles in $\mathcal{M}$ and $\tau\left(I_{\pi}\right)=\pi$. Clearly the correspondence $\pi \rightarrow I_{\pi}$ is effective so there is an $e$ such that $\chi_{e}^{\pi}=I$ for all $\pi \in P(\tau)$. Thus we have proved that $P(\tau) \approx I(\mathcal{M})$.

The correspondence $\pi \rightarrow I_{\pi}$ given in Theorem 3.1 has an additional important property. That is, for any decidable model $\mathcal{M}$ of the theory $T$ constructed as in Theorem 3.1, it is easy to see that the elimination of quantifiers for $T$ ensures that $\operatorname{deg}(\pi)=\operatorname{deg}\left(I_{\pi}\right)=\operatorname{deg}\left(\Gamma\left(I_{\pi}\right)\right)$, where $\Gamma\left(I_{\pi}\right)$ is the $\omega$-type of the set of indiscernibles $I_{\pi}$. Thus, the correspondence $\pi \rightarrow \Gamma\left(I_{\pi}\right)$ proves the following

COROLlary 3.2. Given any infinite recursive binary tree $T$, there is a decidable model $\mathcal{M}$ of an $\omega$-categorical decidable theory $T$ with decidable atoms such that there is an effective 1:1 correspondence between infinite paths through $\tau$ and $\omega$-types of infinite sets of order indiscernibles in $\mathcal{M}$.

In the language of Jockusch and Soare $[\mathbf{4}, \mathbf{5}]$, Corollary 3.2 states that any infinite recursively bounded $\pi_{1}^{0}$ class can be represented as the set of $\omega$-types of infinite sets of order indiscernibles in an $\omega$-categorical decidable model. Our next result will show that the set of $\omega$-types of infinite sets of order indiscernibles in an $\omega$-categorical model is always a recursively bounded $\pi_{1}^{0}$-class as well as complete the proof of our claim that the problem of finding infinite paths through infinite recursive binary trees is equivalent to the problem of finding infinite sets of order indiscernibles in decidable models of $\omega$-categorical decidable theories with decidable atoms.

THEOREM 3.3. For any decidable model $\mathcal{M}$ of an $\omega$-categorical theory $T$ with decidable atoms, there exist an infinite recursive tree $\tau_{\mathcal{M}}$ such that $I(\mid f M) \approx$ $P\left(\tau_{M}\right)$. 
Proof. Fix $\mathcal{M}$ and $T$ as above and let $\rho_{0}, \rho_{1}, \ldots$ be an effective list of all formulas in the language of $T$ such that the free variables of $\rho_{i}$ are among $x_{1}, \ldots, x_{i}$. Given a sequence of 0 's and 1's $\tau=\left\langle\tau_{0}, \ldots, \tau_{n}\right\rangle$, we say that a sequence $\bar{a}=$ $a_{0}, \ldots, a_{n}$ from $\mathcal{M}$ is a sequence of order indiscernibles of type $\tau$ provided that for all $i \leq n$, if $\rho_{i}$ has exactly $k$ free variables, then

(a) $\mathcal{M} \vDash \rho_{i}\left(a_{0}, \ldots, a_{k-1}\right)$ iff $\tau_{i}=1$, and

(b) $\mathcal{M} \models \rho_{i}\left(a_{0}, \ldots, a_{k-1}\right) \Leftrightarrow \rho_{i}\left(a_{\sigma(0)}, \ldots, a_{\sigma(k-1)}\right)$ for all increasing maps $\sigma$ : $\{0, \ldots, k-1\} \rightarrow\{0, \ldots, n\}$.

Clearly there is a formula with exactly $n+1$ free variables in the language of $T$, which we shall denote by $\Psi_{\left\langle\tau_{0}, \ldots, \tau_{n}\right\rangle}$, which says " $x_{1}, \ldots, x_{n}$ is a sequence of order indiscernibles of type $\tau$." Then define the recursive tree $\tau_{\mathcal{M}}$ by letting $\phi \in \tau_{\mathcal{M}}$ and letting $\left\langle\tau_{0}, \ldots, \tau_{k}\right\rangle \in \tau_{\mathcal{M}}$ iff $\mathcal{M} \models \exists x_{0} \ldots \exists x_{k}\left(\Psi_{\left\langle\tau_{0}, \ldots, \tau_{k}\right\rangle}\left(x_{1}, \ldots, x_{k}\right)\right)$.

We claim that $I(\mathcal{M})=P\left(\tau_{\mathcal{M}}\right)$. First, if we are given an infinite set of order indiscernibles $I=\left\langle i_{0}, i_{1}, \ldots\right\rangle$ in $\mathcal{M}$, then let $\pi_{I}\left\langle\tau_{0}, \tau_{1}, \ldots\right\rangle$ be the infinite path through $\tau_{\mathcal{M}}$ where for each $n, i_{0}, \ldots, i_{n}$ is a sequence of order indiscernibles of type $\left\langle\tau_{0}, \ldots, \tau_{n}\right\rangle$. It is easy to see by our definition of $\tau_{\mathcal{M}}$ that $\pi_{I}$ is indeed an infinite path through $\tau_{\mathcal{M}}$ and since $\mathcal{M}$ is decidable, the correspondence $I=\pi_{I}$ is effective. Thus there is an $e$ such that $\chi_{e}^{I}=\pi_{I}$ for all $I \in T(\mathcal{M})$.

Next suppose that $\pi=\left\langle\tau_{0}, \tau_{1}, \ldots\right\rangle$ is an infinite path through $\tau_{\mathcal{M}}$. First we claim that $\left\langle\Psi_{\left\langle\tau_{0}\right\rangle}, \Psi_{\left\langle\tau_{0}, \tau_{1}\right\rangle}, \Psi_{\left\langle\tau_{0}, \tau_{1}, \tau_{2}\right\rangle}, \ldots\right\rangle$ determines an $\omega$-type $\Gamma_{\pi}$ of an infinite set of order indiscernibles in $\mathcal{M}$. That is, $\Gamma_{\pi}$ is complete since $\rho_{n} \in \Gamma_{\pi}$ iff $\tau_{n}=1$ by our definition of $\Psi_{\left\langle\tau_{0}, \ldots, \tau_{n}\right\rangle}$ and $\Gamma_{\pi}$ is consistent since for all $n$,

$$
\mathcal{M} \models \exists x_{0} \cdots \exists x_{n}\left(\Psi_{\left\langle\tau_{0}, \ldots, \tau_{n}\right\rangle}\left(x_{0}, \ldots, x_{n}\right)\right) .
$$

Since $T$ has decidable atoms and $\Gamma_{\pi}$ is an $\omega$-type, we can effectively define from $\pi$ a sequence $k_{0}<k_{1}<\cdots$ such that $\rho_{k_{i}}^{\tau_{i}}$ is an atom of $B_{n_{i}+1}(T)$ and the free variables of $\rho_{k_{i}}$ are exactly $x_{0}, \ldots, x_{n_{i}}$, where $\rho_{j}^{1}=\rho_{j}, \rho_{j}^{0}=\neg \rho_{j}$, and $n_{0}<n_{1}<n_{2}<\cdots$. It follows that if

$$
\theta_{i}=\exists x_{n_{i}+1} \cdots \exists x_{k_{i}}\left(\bigwedge_{j=0}^{k_{i}} \Psi_{\left\langle\tau_{0}, \ldots, \tau_{j}\right\rangle}\right),
$$

then $\theta_{i}$ is also an atom of $B_{n_{i}}(T)$ since $\theta_{i} \rightarrow \rho_{k_{i}}^{\tau_{i}}$ and $\theta_{i}$ is consistent. From the sequence $\left\langle\theta_{0}, \theta_{1}, \ldots\right\rangle$ we can effectively define a sequence $\left\langle a_{0}, a_{1}, \ldots\right\rangle$ of elements of $\mathcal{M}$ as follows:

(i) $a_{0}, \ldots, a_{n_{0}}$ is the sequence with least Gödel number such that

$$
\mathcal{M} \vDash \theta_{0}\left(a_{0}, \ldots, a_{n_{0}}\right) .
$$

(ii) Having defined $a_{0}, \ldots, a_{n_{k}}$, then define $a_{n_{k}+1}, \ldots, a_{n_{k+1}}$ as the sequence with least Gödel number such that $\mathcal{M} \models \theta_{k+1}\left(a_{0}, \ldots, a_{n_{k+1}}\right)$.

The existence of $a_{n_{k}+1}, \ldots, a_{n_{k}}$ is justified as follows. Note that our definition of $\tau_{\mathcal{M}}$ ensures that there exist $b_{0}, \ldots, b_{n_{k+1}}$ such that $\mathcal{M} \vDash \theta_{n_{k+1}}\left(b_{0}, \ldots, b_{n_{k+1}}\right)$. Clearly from our definition of the $\theta_{i}$ 's, $\mathcal{M} \vDash \theta_{n_{k}}\left(b_{0}, \ldots, b_{n_{k}}\right)$ so that $a_{0}, \ldots, a_{n_{k}}$ and $b_{0}, \ldots, b_{n_{k}}$ have the same type since $\theta_{n_{k}}$ is an atom. But since $\mathcal{M}$ is $\omega$ categorical, $\mathcal{M}$ is homogeneous so that there must exist $a_{n_{k}+1}, \ldots, a_{n_{k+1}}$ in $\mathcal{M}$ such that $a_{0}, \ldots, a_{n_{k+1}}$ has the same type as $b_{0}, \ldots, b_{n_{k+1}}$ and hence

$$
\mathcal{M} \models \theta_{n_{k+1}}\left(a_{0}, \ldots, a_{n_{k+1}}\right) \text {. }
$$


Moreover, it is clear that $\left\langle a_{0}, a_{1}, \ldots\right\rangle$ is a sequence of order indiscernibles since the formula $\theta_{n}$ says that $a_{0}, \ldots, a_{n}$ is a sequence of order indiscernibles with respect to $\rho_{0}, \ldots, \rho_{n}$. Thus, if we let $I_{\pi}=\left\langle a_{0}, a_{1}, \ldots\right\rangle$, then we have shown that from $\pi$ we can effectively define $I_{\pi}$. Thus there is an $f$ such that $\chi_{f}^{\pi}=I_{\pi}$ for all $\pi \in P\left(\tau_{\mathcal{M}}\right)$ and, moreover, it is easy to check that $\chi_{e}^{I_{\pi}}=\pi$. Thus $I(\mathcal{M}) \approx P\left(\tau_{\mathcal{M}}\right)$.

We should note that if $T$ is an $\omega$-categorical decidable theory, $T$ does not necessarily have decidable atoms; see [7] for examples. But even if $T$ does not have decidable atoms, our proof of Theorem 3.3 shows that there is an effective correspondence between $\omega$-types of indiscernibles in $\mathcal{M}$ and paths through $T$. That is, the only place in the proof of Theorem 3.3 that we used the fact that $T$ has decidable atoms was to extract from $\pi$ in an effective manner the sequence $\left\langle\theta_{0}, \theta_{1}, \ldots\right\rangle$ of atoms from $\Gamma_{\pi}$. But $\Gamma_{\pi}$ is always an $\omega$-type of an infinite set of order indiscernibles in $\mathcal{M}$, thus the correspondence $\pi \rightarrow \Gamma_{\pi}$ proves the following.

COROLLARY 3.4. For any $\omega$-categorical decidable model $\mathcal{M}$, there is a recursive binary tree $\tau_{\mathcal{M}}$ such that there is an effective $1: 1$ correspondence between the paths through $\tau_{\mathcal{M}}$ and the $\omega$-types of infinite sets of order indiscernibles in $\mathcal{M}$.

Corollaries 3.2 and 3.4 state that the set of degrees realized by the sets of $\omega$-types of infinite sets of order indiscernibles in a decidable $\omega$-categorical model $\mathcal{M}$ coincides with the set of degrees realized by recursively bounded $\pi_{1}^{0}$ classes. Moreover, if the underlying theory of $\mathcal{M}$ has decidable atoms, then from any $\omega$-type $\Gamma$ of an infinite set of order indiscernibles in $\mathcal{M}$, we can effectively construct $I \in I(\mathcal{M})$ of $\omega$-type $\Gamma$. Now Jockusch and Soare have proved many results about the degrees of recursively bounded $\pi_{1}^{0}$-classes in $[\mathbf{4}, \mathbf{5}]$. Thus from Jockusch and Soare's work we immediately derive the following results.

THEOREM 3.5. Let $\mathcal{M}$ be a decidable model of an $\omega$-categorical theory $T$ with decidable atoms.

(i) There is an $I \in I(\mathcal{M})$ such that $(\operatorname{deg}(I))^{\prime}=0^{\prime}$.

(ii) There is an $I \in I(\mathcal{M})$ such that $\operatorname{deg}(I)$ is an r.e. degree.

(iii) If there is no recursive infinite set of order indiscernibles in $\mathcal{M}$ and $\bar{a}_{0}, \bar{a}_{1}, \ldots$ is any countable sequence of nonzero degrees, then there are a continuum of $\omega$-types of infinite sets of order indiscernibles in $\mathcal{M}, C$, which have mutually incomparable degrees and which are all incomparable to any $a_{i}$.

THEOREM 3.6. (A) There exist decidable models $\mathcal{M}_{1}, \mathcal{M}_{2}, \mathcal{M}_{3}$ of an $\omega$-categorical theory with decidable atoms such that:

(i) $\mathcal{M}_{1}$ has no recursive $I \in I(\mathcal{M})$ but there is $I^{\prime} \in I(\mathcal{M})$ which is of a minimal degree.

(ii) $\mathcal{M}_{2}$ has $\omega$ recursive $\omega$-types of infinite sets of order indiscernibles and exactly one nonrecursive $\omega$-type of an infinite set of order indiscernibles.

(iii) $\mathcal{M}_{3}$ has infinitely many $\omega$-types of infinite sets of order indiscernibles, all of which are mutually Turing incomparable.

(B) For any r.e. deyree $d$, there exists an $\mathcal{M}_{d}$ such that the degrees of $\omega$-types of infinite sets of order indiscernibles consist of all r.e. degrees $\geq_{T} d$.

Finally, we observe that given an $\omega$-categorical decidable theory $T$, we can always decide whether a formula $\rho\left(x_{1}, \ldots, x_{n}\right)$ is an atom in $B_{n}(T)$ effectively in $0^{\prime}$. That 
is, if $\rho_{0}^{n}, \rho_{1}^{n}, \ldots$ is an effective list of the formulas of $T$ with $n$ free variables, then $\rho\left(x_{1}, \ldots, x_{n}\right)$ is an atom in $B_{n}(T)$ iff the r.e. set

$$
\begin{aligned}
E_{\rho}=\left\{i \mid T \models\left(\forall x_{1} \cdots \forall x_{n}\left(\rho_{i}^{n}\left(x_{1}, \ldots, x_{n}\right) \rightarrow \rho\left(x_{1}, \ldots, x_{n}\right)\right)\right)\right. \\
\left.\&\left(\neg \forall x_{1} \cdots \forall x_{n}\left(\rho\left(x_{1}, \ldots, x_{n}\right) \rightarrow \rho_{i}^{n}\left(x_{1}, \ldots, x_{n}\right)\right)\right)\right\}
\end{aligned}
$$

is empty and the question of whether $E_{\rho}$ is empty can be answered recursively in $0^{\prime}$. Thus, in the proof of Theorem 3.3, one can easily check that for any path $\pi \in \tau_{\mathcal{M}}, I_{\pi}$ is recursive in the join of the degree of $\pi$ and $0^{\prime}$ even if the decidable $\omega$-categorical theory $T$ failed to have decidable atoms. Now Jockusch and Soare in [5] proved that in any recursive binary tree $\tau$ with an infinite path, there exists an infinite path $\pi \in P(\tau)$ such that the jump of $\pi$ is $0^{\prime}$. Thus, if we start with a decidable $\omega$-categorical model $\mathcal{M}$ and construct the recursive binary tree $\tau_{\mathcal{M}}$ as in Theorem 3.3, then for the path $\pi \in P\left(\tau_{\mathcal{M}}\right)$ such that $\operatorname{deg}(\pi)^{\prime}=0^{\prime}$, we know that $I_{\pi}$ is recursive in $0^{\prime}$. Thus in contrast to the example of a decidable model $\mathcal{M}^{\prime}$ which has infinite sets of order indiscernibles but has no hyperarithmetic infinite sets of order indiscernibles in Corollary 2.7, we can prove the following for $\omega$-categorical decidable models in general.

THEOREM 3.7. If $\mathcal{M}$ is an $\omega$-categorical decidable model, then $\mathcal{M}$ has an infinite set of order indiscernibles $I$ such that $\operatorname{deg}(I) \leq_{T} 0^{\prime}$.

We end this paper with an obvious question in light of Theorem 3.7 and Theorem 3.5(i). Namely, for an arbitrary $\omega$-categorical model $\mathcal{M}$, is Theorem 3.7 the best possible result or can we always find infinite sets of order indiscernibles $I$ in $\mathcal{M}$ such that $\operatorname{deg}(I)^{\prime}=0^{\prime}$ as in the case of decidable $\omega$-categorical theories with decidable atoms?

\section{REFERENCES}

1. C. C. Chang and H. J. Keisler, Model theory, North-Holland, Amsterdam, 1973.

2. A. Ehrenfeucht and A. Mostowski, Models of axiomatic theories admitting automorphisms, Fund. Math. 43 (1956), 50-68.

3. L. A. Harrington and A. S. Kechris, $A$ basis result for $\Sigma_{3}^{0}$ sets of reals with an application to minimal covers, Proc. Amer. Math. Soc. 53 (1975), 445-448.

4. C. G. Jockusch and R. I. Soare, Degrees of members of $\pi_{1}^{0}$ classes, Pacific J. Math. 40 (1972), 605-616.

5. __ $\pi_{1}^{0}$ classes and degrees of theories, Trans. Amer. Math. Soc. 173 (1972), 33-56.

6. H. A. Kierstead and J. B. Remmel, Indiscernibles and decidable models, J. Symbolic Logic 48 (1983), 21-32.

7. J. Schmerl, A decidable $\omega$-categorical theory with a non-recursive Ryall-Nardzewski function, Fund. Math. 98 (1978), 121-125.

8. H. J. Rogers, Jr., Theory of recursive functions and effective computability, McGraw-Hill, New York, 1967.

Department of Mathematics and Statistics, University of South Carolina, Columbia, SOUTh CAROLINA 29208

Department of Mathematics, University of California at San Diego, La JOLLA, CALIFORNIA 92093 